\title{
Phase I/II study of Resiquimod as an immunologic adjuvant for NY-ESO-1 protein vaccination in patients with melanoma
}

\author{
Rachel L Sabado ${ }^{1 *}$, Anna Pavlick², Sacha Gnjatic ${ }^{1,3}$, Crystal Cruz², Isabelita Vengco ${ }^{2}$, Farah Hasan', \\ Farbod Darvishian², Luis Chiriboga ${ }^{2}$, Rose Marie Holman', Juliet Escalon², Caroline Muren², Crystal Escano², \\ Ethel Yepes ${ }^{2}$, Dunbar Sharpe ${ }^{2}$, Sylvia Adams², Patrick Ott ${ }^{2}$, Achim Jungbluth ${ }^{3}$, Linda Pan ${ }^{3}$, Ralph Venhaus ${ }^{3}$, \\ Nina Bhardwaj, ${ }^{1,2}$
}

From Society for Immunotherapy of Cancer 28th Annual Meeting National Harbor, MD, USA. 8-10 November 2013

\section{Purpose}

The TLR 7/8 agonist, Resiquimod has been shown to induce local activation of immune cells, production of cytokines, and antigen-presentation by dendritic cells, features desirable for cancer vaccine adjuvants. In this study, we evaluated the safety and immunogenicity of vaccination with NY-ESO-1 protein emulsified in Montanide ISA-51 VG when given with or without Resiquimod in surgically resected stage IIB-IV melanoma patients.

\section{Experimental design}

This is a two-part study design. Part I represents an open-label dose-escalation with Resiquimod using 2 cohorts treated with $100 \mu \mathrm{g}$ NY-ESO-1 protein emulsified in $1.25 \mathrm{~mL}$ Montanide (day1) followed by topical application of $1000 \mathrm{mg}$ of the $0.2 \%$ Resiquimod gel on days 1 and 3 for cohort- $1(\mathrm{~N}=3)$ or days 1,3 , and 5 for cohort-2 $(\mathrm{N}=3)$. The cycles were repeated every 3 weeks, total of 4 cycles. For part II of the study, patients were blindly randomized to receive $100 \mu \mathrm{g}$ NY-ESO-1 protein emulsified in $1.25 \mathrm{~mL}$ Montanide (day1) followed by topical application of placebo gel (Arm-A; $N=8)$ or $1000 \mathrm{mg}$ of $0.2 \%$ Resiquimod gel (Arm-B; $\mathrm{N}=12$ ) using the dosing regimen established in Part I. Blood samples were collected at baseline, one week after each cycle of vaccination, and at follow-up visit for the assessment of NY-ESO-1-specific humoral and cellular immune responses.

${ }^{1}$ New York University School of Medicine, New York, NY, USA

Full list of author information is available at the end of the article

\section{Results}

The vaccines were generally well-tolerated, with no grade 4 adverse events or study-related deaths. Most study participants experienced mild adverse reactions reported as Grade 1 or 2 per CTCAE criteria v. 4 . One patient experienced a grade 3 syncopal episode that was unrelated to the study drugs and another patient had a grade 3 injection site necrosis that was possibly related to the study drugs. NY-ESO-1 specific antibody responses were induced in both study arms although higher mean antibody titers were observed in Arm B. NY-ESO-1 specific $\mathrm{CD} 4+\mathrm{T}$ cell responses were induced in patients in both study arms. However, significant NY-ESO-1 CD8+ T cell responses were detected only in Arm B.

\section{Conclusions}

The current study shows that Resiquimod is safe and contributes to the induction of immune responses in patients.

\section{Authors' details}

${ }^{1}$ New York University School of Medicine, New York, NY, USA. ${ }^{2}$ Mount Sinai Icahn School of Medicine, New York, NY, USA. ${ }^{3}$ Ludwig Institute for Cancer Research, New York, NY, USA.

Published: 7 November 2013

\section{doi:10.1186/2051-1426-1-S1-P272}

Cite this article as: Sabado et al:: Phase I/II study of Resiquimod as an immunologic adjuvant for NY-ESO-1 protein vaccination in patients with melanoma. Journal for ImmunoTherapy of Cancer 2013 1(Suppl 1): P272. 